\title{
Standard Neoadjuvant Treatment in Early/Locally Advanced Breast Cancer
}

\author{
Cristina Pernaut Flora Lopez Eva Ciruelos \\ Breast Cancer Unit, Medical Oncology Department, University Hospital 12 de Octubre, Madrid, Spain
}

\section{Keywords \\ Breast cancer - Neoadjuvant treatment . Pathological complete response}

\section{Summary}

Neoadjuvant treatment allows us to improve surgical results and test new drugs. In recent years, there have been significant advances in the field of neoadjuvant treatment, including hormonal neoadjuvant therapy in luminal tumors, double blockade in HER2-positive tumors, and the use of platinum salts in triple-negative tumors.

(C) 2018 S. Karger GmbH, Freiburg

\section{Introduction}

In early breast cancer, surgery is the mainstay of curative treatment. Complementary local (radiotherapy) and systemic (adjuvant endocrine therapy or chemotherapy) treatments are associated with the aim of reducing the risk of relapse according to the clinicopathological characteristics of the tumor. However, the possibility of administering these therapies prior to surgery (neoadjuvant setting) offers several advantages: reduction in tumor size to improve respectability, an increased rate of conservative surgery improving esthetic results, reduction in the extent of axillary surgery, and early treatment of micrometastatic disease.

Two main clinical trials have compared the administration of chemotherapy before or after surgery in operable breast cancer, NSABP B-18 [1] and NSABP B-27 [2, 3]. Both demonstrate that preoperative therapy is equivalent to adjuvant therapy in the shortand long-term outcomes. B-27 also showed that the addition of preoperative taxanes to anthracyclines improves response. The design and results of these studies are detailed in table 1.
Moreover, with neoadjuvant treatment, we can provide information about the tumor's behavior in vivo and obtain prognostic factors such as pathologic complete response (pCR) or residual cancer burden (RCB) without compromising survival [4].

In general, the indications for neoadjuvant therapy according to clinical practice guidelines are:

- Locally advanced tumors (stage IIB-IIIC).

- Selected cases with early-stage disease:

- Unfavorable tumor/breast size ratio

- Specific subtypes with high response rates (triple-negative and HER2-positive)

- Attempted axillary downstaging

- Patients with temporary contraindications for surgery

To comply with all these conditions, it is necessary to have a multidisciplinary team in which each case is discussed.

\section{Evaluation of Response to Neoadjuvant Treatment}

The radiologic evaluation before the start of treatment is discussed in the article on surgical management by Cordoba et al. [5] in this focus of BREAST CARE [6].

During treatment, clinical response has to be assessed by physical examination. Imaging tests will be requested if progressive disease is suspected. At the end of treatment, radiologic re-evaluation with magnetic resonance imaging (MRI) is recommended with the exception of patients who are candidates for mastectomy where the surgical indication does not change. In those patients, performing a prior MRI study is optional, with the awareness that MRI will not be useful to assess response to treatment but only provide anatomical information before surgery and that it has a high false-positive rate.

After surgery, we have 2 histologic response markers that have an important role as prognostic factors: pCR and RCB. pCR has been inconsistently defined in different clinical trials; a recent pooled analysis of neoadjuvant trials [7] concluded that the preferred definition of pCR is the eradication of invasive tumor cells

\section{KARGER}

() 2018 S. Karger GmbH, Freiburg

Fax +497614520714 
Table 1. NSABP B-18 and NSABP B-27 trials

\begin{tabular}{llllll}
\hline NSABP & $\mathrm{n}$ & Groups & $\mathrm{pCR}, \%$ & $\mathrm{DFS}$ & OS \\
\hline B-18 & \multirow{2}{*}{1,523} & 1. surgery $\rightarrow$ AC $\times 4$ vs. & 13 & HR 0.98 & HR 0.99 \\
& & 2. AC $\times 4 \rightarrow$ surgery & $\mathrm{p}=0.78$ & $\mathrm{p}=0.9$ \\
\hline B-27 & \multirow{2}{*}{2,344} & 1. AC $\times 4 \rightarrow$ surgery vs. & 12.8 & HR $0.93-0.92$ & HR 0.93-0.97 \\
& & 2. AC $\times 4 \rightarrow$ D $\times 4 \rightarrow$ surgery vs. & 26.1 & $\mathrm{p}=0.29$ & $\mathrm{p}=0.46-0.76$ \\
& & 3. AC $\times 4 \rightarrow$ surgery $\rightarrow$ D $\times 4$ & 14.3 & & \\
\hline
\end{tabular}

$\mathrm{pCR}=$ Pathological complete response; $\mathrm{DFS}=$ disease-free survival; $\mathrm{OS}=$ overall survival;

$\mathrm{AC}=$ doxorubicin + cyclophosphamide; $\mathrm{D}=$ docetaxel; $\mathrm{HR}=$ hazard ratio.

from both breast and lymph nodes (ypT0 ypN0 or ypTis ypN0) as this is better associated with improved event-free survival (EFS) and overall survival (OS) than other prior pCR definitions (tumor eradication from breast only ypT0).

Furthermore, the association with better long-term outcomes is stronger in more aggressive tumor types (triple-negative, HER2positive/hormone receptor(HR)-negative, high-grade HR-positive).

Another parameter that provides us with information about tumor behavior is the RCB, a value calculated from the residual tumor characteristics and which grades the pathological response from 0 (without residual disease) to III (extensive residual disease). It is an independent prognostic factor that also correlates with disease-free survival (DFS) by breast cancer subtype in a similar manner as PCR [8].

\section{Endocrine Therapy}

Several studies have attempted to explore the role of hormonal therapy in the setting of neoadjuvant treatment of breast cancer. As the response of luminal tumors to endocrine treatment is biologically different, it is reasonable to employ an alternative parameter to pCR that evaluates the response to treatment and its correlation with prognosis. This tool is the preoperative endocrine prognostic index (PEPI) that provides a score based on the sum of risk points derived from $\mathrm{pT}$ stage, $\mathrm{pN}$ stage, ki67 level, and estrogen receptor (ER) status of the surgical specimen. The PEPI score provides a prognostic value to aid the decision regarding subsequent adjuvant treatment [9].

On the other hand, it is important to highlight the information obtained from the reduction in the expression of the proliferation antigen Ki-67 during hormonal treatment as a response marker and as a surrogate prognostic marker, when compared with Ki-67 at baseline. A subanalysis of 158 patients from the IMPACT trial observed that a high Ki-67 value after 2 weeks of endocrine therapy was correlated with a worse recurrence-free survival, while higher Ki-67 expression at baseline was not [10]. These data suggest that the measurement of changes in Ki-67 after neoadjuvant therapy is a better predictor of prognosis than the baseline value.

This hypothesis was confirmed in a recent study (POETIC trial [11]) with a larger number of patients $(n=4,480)$ in whom ki67 was measured at baseline and after 2 weeks of neoadjuvant endocrine therapy; both results were stratified into low $(<10 \%)$ or high
Table 2. Main trials in neoadjuvant endocrine therapy

\begin{tabular}{lllll}
\hline Trial & $\mathrm{n}$ & Groups & CRR, \% & BCS rate, \% \\
\hline P024 & \multirow{2}{*}{337} & T 4 months & 36 & 35 \\
& & L 4 months & 55 & 45 \\
& & $\mathrm{p}<0.001$ & $\mathrm{p}=0.022$ \\
\hline IMPACT & \multirow{2}{*}{330} & T 12 weeks & 36 & 31 \\
& & A 12 weeks & 37 & 44 \\
& & A+T 12 weeks & 39 & 24 \\
\hline PROACT & \multirow{2}{*}{ 451 } & T 3 months & 35.4 & 30.8 \\
& & A 3 months & 39.5 & 43 \\
\hline ACOSOG Z-1031 & \multirow{2}{*}{374} & E 16-18 weeks & 62.9 & 67.8 \\
& & L 16-18 weeks & 74.8 & 60.8 \\
& & A 16-18 weeks & 69.1 & 77 \\
\hline
\end{tabular}

$\mathrm{T}=$ Tamoxifen $; \mathrm{L}=$ letrozole; $\mathrm{E}=$ exemestane; $\mathrm{CRR}=$ clinical response rate; $\mathrm{BCS}=$ breast-conserving surgery.

(>10\%) expression. Low Ki-67 at baseline was correlated with a better prognosis and no need for other systemic treatment. In contrast, a high Ki-67 value after 2 weeks of hormonal treatment was found to be a bad prognostic factor suggesting the need for adjuvant chemotherapy. However, baseline Ki-67 is not a good indicator to identify luminal subtype (LumA/LumB) because of the low correlation with PAM50 intrinsic subtypes (3.3\% HER2-enriched and basal-like) [12].

\section{Tamoxifen versus Aromatase Inhibitors}

Greater responses in terms of breast-conserving surgery (BCS) are obtained with aromatase inhibitors (AI) versus tamoxifen in postmenopausal women with ER-positive/HER2-negative breast cancer (table 2) [13]. However, no statistically significant differences were found when exemestane, letrozol, and anastrozole were compared [12], obtaining a clinical response rate higher than $60 \%$ with all $3 \mathrm{AI}$. After these results, on the basis of slightly higher clinical response rates, letrozole and anastrozole were selected for further investigation, and no other differences in surgical outcome, PEPI score, or Ki-67 suppression were detected. The BCS rate for mastectomy candidates at diagnosis was $51 \%$.

Regarding the optimal duration of endocrine neoadjuvant therapy, in all previous studies, treatment was administered for approximately 4 months (12-16 weeks) with an interim analysis of Ki-67 at 2-4 weeks to check response. 
In terms of effectiveness, a greater benefit in biological parameters was observed in LumA subtypes according to PAM50 [12], and no responses were detected in the non-luminal subtypes (3.3\%). In a small subgroup of LumA patients, a Ki-67 increase was observed that seems to be related to hormone resistance mechanisms.

With these data, best candidates for endocrine neoadjuvant therapy will be those who are postmenopausal with an intrinsic LumA subtype, elderly, or unfit for chemotherapy.

In the ACOSOG trial [12], the subgroup with Ki-67 > 10\% after 2 weeks of endocrine therapy had worse recurrence-free survival despite the fact that most patients $(71 \%)$ completed neoadjuvant chemotherapy.

\section{Chemotherapy and Antiangiogenic Agents}

Chemotherapy, given with anti-HER2-targeted drugs in the case of HER2-positive disease, is the standard neoadjuvant approach. Commonly used regimens for patients with HER2-negative disease, especially in the higher-risk category, include anthracycline-based regimens such as doxorubicin and cyclophosphamide followed or proceeded by a taxane (docetaxel or paclitaxel). For those in whom the potential cardiotoxic effects of anthracyclines are a primary concern, non-anthracycline regimens are a reasonable alternative [14].

\section{Anthracycline-Based Regimens}

The rationale for the use of anthracyclines and taxanes in the preoperative setting comes directly from clinical trials of neoadjuvant treatment [15]. Specifically in the neoadjuvant setting, multiple studies have demonstrated that the addition of a taxane to an anthracycline-based regimen, either concurrently or sequentially, is associated with increased response rates (table 1).

\section{Neoadjuvant Carboplatin and Bevacizumab}

The hypothesis of an increased efficacy of platinum agents in triple-negative breast cancer (TNBC) is based on the fact that these tumors often show functional DNA repair alterations that increase the sensitivity to cross-linking agents [16].

The phase II GeparSixto trial [16] explored the impact of the addition of carboplatin to neoadjuvant chemotherapy based in anthracyclines and taxanes. 595 patients with previously untreated, non-metastatic, stage II-III TNBC and HER2-positive breast cancer were enrolled. Patients were treated with concurrent weekly paclitaxel and weekly non-pegylated liposomal doxorubicin. $\mathrm{Pa}-$ tients with TNBC received simultaneous bevacizumab, and patients with HER2-positive disease received simultaneous trastuzumab and lapatinib. Patients were randomly assigned at a 1:1 ratio to receive concurrent carboplatin or not. 43.7 versus $36.9 \%$ achieved a pCR, favoring treatment with carboplatin. However, this regimen seems to significantly increase responses in patients with TNBC (53.2 vs. $36.9 \%$; $\mathrm{p}=0.005)$, but not in those with HER2-positive disease ( 32.8 vs. $36.8 \%$; $\mathrm{p}=0.581$ ).
The phase II trial CALGB 40603 (Alliance) [17] explored the combination of carboplatin and/or bevacizumab with neoadjuvant chemotherapy. 443 patients with stage II-III TNBC received weekly paclitaxel followed by doxorubicin plus cyclophosphamide every 2 weeks (ddAC), and were randomly assigned to concurrent carboplatin and/or bevacizumab (in a $2 \times 2$ factorial design).

Only the addition of carboplatin ( 54 vs. $41 \%$; $\mathrm{p}=0.0029$ ) significantly raised pCR (defined as ypT0/is ypN0). The increase in the pCR breast/axilla rate with bevacizumab (52 vs. $44 \%$ ) did not achieve statistical significance $(\mathrm{p}=0.057)$. Again, patients who received both agents had the highest pCR rate $(60 \%)$ with no significant interaction between their effects $(\mathrm{p}=0.43)$. More-than-additive interactions between the 2 agents could not be demonstrated.

Patients assigned to either carboplatin or bevacizumab were less likely to complete weekly paclitaxel and ddAC without skipped doses, dose modification, or early discontinuation resulting from toxicity.

The phase III NSABP-40 trial [18] explored the addition of bevacizumab. This trial had a $3 \times 2$ factorial design, and enrolled patients with operable, HER2-non-amplified breast cancer, clinical stage T1c-3, cN0, cN1, or cN2a. Patients received 1 of 3 docetaxelbased neoadjuvant regimens: docetaxel alone, docetaxel plus capecitabine, or docetaxel plus gemcitabine. These 3 taxane arms were followed by neoadjuvant doxorubicin and cyclophosphamide. Those groups were randomly assigned (1:1:1:1:1:1) to receive bevacizumab (neoadjuvant and adjuvant settings) versus not.

The addition of capecitabine or gemcitabine to docetaxel therapy, as compared with docetaxel therapy alone, did not significantly increase the rate of pCR (23.2 and $26.9 \%$ vs. $25.8 \%$; p = 0.51 ), and both were associated with increased toxicity. The addition of bevacizumab did not significantly increase the rate of pCR (defined as ypT0 ypN0) (27.6 vs. $23 \%$; p = 0.08).

The addition of carboplatin increased hematologic and non-hematologic toxicity, with subsequent dose reductions and discontinuations ( $48 \%$ with carboplatin and $39 \%$ without carboplatin; $\mathrm{p}=$ 0.031) [18]. Hematologic and non-hematologic toxic effects were significantly more common in the carboplatin group than in the no-carboplatin group. This increased toxicity and the lack of clear long-term survival benefits are the main reasons why the use of platinum agents is controversial as standard care for TNBC. In spite of this, the evidence that PCR is strongly associated with OS in TNBC is an argument often used by physicians to justify the addition of carboplatin to neoadjuvant systemic treatment in clinical practice. Several phase III trials are ongoing and may provide more information on this topic.

The use of bevacizumab in the neoadjuvant setting is still controversial and is not recommended, mainly due to the lack of survival benefit evidence and the increase in toxicity.

\section{Neoadjuvant Nab-Paclitaxel}

In the phase III GeparSepto trial [19], 1,026 patients (396 HER2-positive, 276 triple-negative, and 534 HR-positive/HER2negative) with previously untreated unilateral or bilateral breast cancer were randomized 1:1 to receive weekly nab-paclitaxel 150 
Table 3. Main studies of neoadjuvant chemotherapy

\begin{tabular}{|c|c|c|c|c|}
\hline Study [ref.] & $\mathrm{n}$ & $\begin{array}{l}\text { Breast cancer } \\
\text { subtypes }\end{array}$ & Treatment & pCR rate, $\%$ \\
\hline \multicolumn{5}{|c|}{ Anthracyclines and taxanes } \\
\hline NSABP B-27 [15] & 2,344 & all & AC vs. AC $\rightarrow$ docetaxel & 13 vs. $26(\mathrm{p}<0.0001)$ \\
\hline \multicolumn{5}{|c|}{ Carboplatin and bevacizumab } \\
\hline GeparSixto [16] & 595 & $\begin{array}{l}315 \text { TNBC } \\
273 \text { HER2+ }\end{array}$ & $\begin{array}{l}\text { paclitaxel }+ \text { non-pegilated doxorubicin }+ \\
\text { bevacizumab or lapatinib }+ \text { trastuzumab } \\
\pm \text { carboplatin }\end{array}$ & 43.7 vs. $36.9(\mathrm{p}=0.107)$ \\
\hline CALGB 40603 [17] & 443 & TNBC & $\begin{array}{l}\text { paclitaxel } \rightarrow \mathrm{ddAC} \pm \text { carboplatin and/or } \\
\text { bevacizumab }\end{array}$ & $\begin{array}{l}\text { carboplatin } 54 \text { vs. } 41 \\
(\mathrm{p}=0.0029) \\
\text { bevacizumab } 52 \text { vs. } 44 \\
(\mathrm{p}=0.057)\end{array}$ \\
\hline NSABP-40 [18] & 1,206 & $\begin{array}{l}479 \text { TNBC } \\
707 \text { luminal }\end{array}$ & $\begin{array}{l}\text { docetaxel } \rightarrow \mathrm{AC} \pm \text { bevacizumab } \\
\text { docetaxel }+ \text { capecitabine } \rightarrow \mathrm{AC} \pm \\
\text { bevacizumab } \\
\text { docetaxel }+ \text { gemcitabine } \rightarrow \mathrm{AC} \pm \\
\text { bevacizumab }\end{array}$ & 27.6 vs. $23(\mathrm{p}=0.08)$ \\
\hline
\end{tabular}

\begin{tabular}{|c|c|c|c|c|}
\hline \multicolumn{5}{|l|}{ NAB-paclitaxel } \\
\hline GeparSepto [19] & 1,229 & $\begin{array}{l}396 \text { HER2+ } \\
276 \text { TNBC } \\
534 \text { HR+/HER2- }\end{array}$ & $\begin{array}{l}\text { nab-paclitaxel vs. paclitaxel } \rightarrow \mathrm{EC} \\
\text { HER2+: trastuzumab and pertuzumab }\end{array}$ & 38 vs. $29(\mathrm{p}=0.00065)$ \\
\hline $\begin{array}{l}\text { WSG-ADAPT-TN } \\
{[21]}\end{array}$ & 336 & TNBC & $\begin{array}{l}\text { nab-paclitaxel + carboplatin vs. } \\
\text { nab-paclitaxel + gemcitabine }\end{array}$ & 45.9 vs. $28.7(p=0.002)$ \\
\hline
\end{tabular}

$\mathrm{TNBC}=$ Triple-negative breast cancer; $\mathrm{HR}=$ hormone receptor; $\mathrm{AC}=$ doxorubicin + cyclophosphamide; $\mathrm{ddAC}=\mathrm{AC}$ every 2 weeks; $\mathrm{EC}=$ epirubicin + cyclophosphamide $\mathrm{mg} / \mathrm{m}^{2}$ (after study amendment $125 \mathrm{mg} / \mathrm{m}^{2}$ due to the higher incidence of severe sensory neuropathy) or conventional paclitaxel (80 $\mathrm{mg} / \mathrm{m}^{2}$ [2] weekly), followed by epirubicin plus cyclophosphamide. Patients with HER2-positive tumors received concurrent trastuzumab and pertuzumab.

pCR was higher in the nab-paclitaxel group (38\%) than in the paclitaxel group $(29 \%)(p=0.00065)$. In the efficacy analysis by subgroup, significant differences were observed in the TNBC subtype ( 48 vs. $26 \% ; \mathrm{p}=0.00027$ ). No significant differences were observed either in patients with HER2-positive tumors (62 vs. 54\%; p $=0.13$ ) or in those with HER2-negative/HR-positive disease (16 vs. $12 \% ; \mathrm{p}=0.23$ ). However, in the HER2-negative/HR-positive group, a benefit in terms of DFS after a median follow-up of 4 years (hazard ratio $0.71 ; \mathrm{p}=0.660$ ) was observed, similar to the benefit in the TNBC group (hazard ratio $0.66 ; \mathrm{p}=0.0694$ ) [20].

\section{Neoadjuvant Nab-Paclitaxel Combined with Carboplatin}

In the WSG-ADAPT-TN trial [21], 336 TNBC patients were randomly assigned to nab-paclitaxel plus gemcitabine (arm A) versus nab-paclitaxel plus carboplatin (arm B), in an anthracyclinefree regimen. pCR favored arm B (28.7 vs. $45.9 \%$; $p=0.002)$, which shows the high efficacy and excellent tolerability of a neoadjuvant nab-paclitaxel plus carboplatin regimen in TNBC. The authors concluded that the de-escalation of further chemotherapy in patients with early pCR after a short anthracycline-free regimen is a promising field of future research (table 3 ).

\section{Anti-HER2 Agents}

\section{Neoadjuvant Trastuzumab}

Molecular targeting of the HER2 receptor with the humanized monoclonal antibody trastuzumab improved DFS and OS in patients with both metastatic and early HER2-positive breast cancer [22].

The phase III NOAH trial [23] explored the addition of trastuzumab to neoadjuvant chemotherapy. 235 patients with HER2positive locally advanced or inflammatory breast cancer were randomly assigned 1:1 to receive chemotherapy alone (consisting of doxorubicin, paclitaxel, cyclophosphamide, methotrexate, and fluorouracil) or with 1 year of trastuzumab (concurrently with neoadjuvant chemotherapy and continued after surgery). A parallel cohort of 99 patients with HER2-negative disease was included and treated with the same chemotherapy regimen.

The rate of pCR was 19\% in the arm without trastuzumab and $38 \%$ in the arm with trastuzumab $(\mathrm{p}=0.001)$. The rate of $\mathrm{pCR}$ in the HER2-negative arm was $16 \%$.

The 5-year EFS was 58\% (95\% confidence interval (CI) 48-66) in the trastuzumab group and $43 \%$ (95\% CI $34-52)$ in the chemotherapy group (hazard ratio $0.64,95 \%$ CI $0.44-0.93$; two-sided logrank $p=0.016)$. EFS was strongly associated with $p C R$ in patients who received trastuzumab. Of the 68 patients with a PCR, the hazard ratio for EFS between those with and without trastuzumab was 0.29 (95\% CI 0.11-0.78). Results showing improved survival in the trastuzumab group were not significant in this trial, probably be- 
Table 4. Main studies of anti-HER2 agents

\begin{tabular}{|c|c|c|c|c|c|}
\hline Study [ref.] & $\mathrm{n}$ & Neoadjuvant chemotherapy & Neoadjuvant anti-HER2 agent & Adjuvant therapy & $\mathrm{pCR}, \%$ \\
\hline NOAH [23] & 235 & $\mathrm{AP} \rightarrow$ paclitaxel $\rightarrow \mathrm{CMF}$ & $\begin{array}{l}\text { trastuzumab } \\
-\end{array}$ & trastuzumab & $\begin{array}{l}38 \\
19\end{array}$ \\
\hline GeparQuinto [25] & 615 & $\mathrm{EC} \rightarrow$ docetaxel & $\begin{array}{l}\text { trastuzumab } \\
\text { lapatinib }\end{array}$ & trastuzumab & $\begin{array}{l}22.7 \\
30.3\end{array}$ \\
\hline NeoALTTO [26] & 455 & paclitaxel & $\begin{array}{l}\text { trastuzumab } \\
\text { lapatinib } \\
\text { trastuzumab + lapatinib }\end{array}$ & $\begin{array}{l}\text { trastuzumab + FEC } \\
\text { lapatinib + FEC } \\
\text { trastuzumab + lapatinib + FEC }\end{array}$ & $\begin{array}{l}27.6 \\
20 \\
46.8\end{array}$ \\
\hline NeoSphere [27] & 417 & $\begin{array}{l}\text { docetaxel } \\
\text { docetaxel } \\
- \\
\text { docetaxel }\end{array}$ & $\begin{array}{l}\text { trastuzumab } \\
\text { trastuzumab + pertuzumab } \\
\text { trastuzumab + pertuzumab } \\
\text { pertuzumab }\end{array}$ & $\begin{array}{l}\text { trastuzumab }+ \text { FEC } \\
\text { trastuzumab }+ \text { FEC } \\
\text { trastuzumab + docetaxel } \rightarrow \text { FEC } \\
\text { trastuzumab + FEC }\end{array}$ & $\begin{array}{l}29 \\
45.8 \\
16.8 \\
24\end{array}$ \\
\hline TRYPHAENA [28] & 225 & $\begin{array}{l}\mathrm{FEC} \rightarrow \text { docetaxel } \\
\text { FEC } \rightarrow \text { docetaxel } \\
\text { docetaxel }+ \text { carboplatin }\end{array}$ & trastuzumab + pertuzumab & trastuzumab & $\begin{array}{l}50.7 \\
45.3 \\
51.9\end{array}$ \\
\hline
\end{tabular}

pCR = Pathologic complete response; $\mathrm{AP}$ = doxorubicin + cisplatin; $\mathrm{CMF}=$ cyclophosphamide, methotrexate, 5 -fluorouracil; EC = epirubicin + cyclophosphamide; $\mathrm{FEC}=5$-fluorouracil, epirubicin, cyclophosphamide.

cause of a crossover to adjuvant trastuzumab in $17 \%$ of patients and the use of trastuzumab at relapse.

The phase III Z1041 trial [24] explored the concurrent administration of trastuzumab with anthracyclines. 282 patients with HER2-positive breast cancer were randomly assigned (1:1) to sequential treatment (5-fluorouracil, epirubicin, cyclophosphamide (FEC) followed by weekly paclitaxel and trastuzumab) or to concurrent treatment (paclitaxel and trastuzumab followed by FEC-75 and weekly trastuzumab). There was not additional benefit of the concurrent treatment in terms of pCR (defined in this trial as ypT0; 56.6 vs. $54.2 \%)$.

\section{Dual Blockade with Trastuzumab plus Lapatinib}

In the phase III GeparQuinto trial [25], 620 patients with untreated HER2-positive operable or locally advanced breast cancer were randomly assigned at a 1:1 ratio to receive neoadjuvant treatment with epirubicin plus cyclophosphamide followed by docetaxel, with either trastuzumab or lapatinib. Patients completed post-surgery trastuzumab treatment for 1 year in both treatment groups. The rate of pCR was lower in the lapatinib arm (22.7 vs. $30.3 \%$ in the trastuzumab arm), and the authors concluded that lapatinib should not be used outside of clinical trials as single antiHER2 treatment in combination with neoadjuvant chemotherapy.

In the phase II NeoAltto trial [26], 529 patients with operable HER2-positive tumors were randomly assigned 1:1:1 to lapatinib, trastuzumab, or lapatinib plus trastuzumab for 6 weeks, followed by an additional 12 weeks of the assigned anti-HER 2 therapy in combination with weekly paclitaxel $\left(80 \mathrm{mg} / \mathrm{m}^{2}\right)$. After surgery, women received FEC followed by 34 weeks of the same assigned neoadjuvant anti-HER2 therapy. pCR (breast and axilla) was achieved in $20 \%$ of the patients in the lapatinib arm, $27.6 \%$ in the trastuzumab arm $(\mathrm{p}=0.13)$, and $46.8 \%$ in the combination group ( $\mathrm{p}=0.0007$ ). However, lapatinib plus trastuzumab did not significantly improve DFS compared with trastuzumab alone (hazard ratio $0.84,97.5 \%$ CI $0.70-1.02 ; \mathrm{p}=0.048$ ( $\mathrm{p} \leq 0.025$ required for statistical significance to adjust for multiple testing). Additionally, this combination has a higher rate of toxicities (such as diarrhea, rash, and hepatic toxicity) and a higher rate of interruption of the neoadjuvant treatment due to adverse events.

\section{Dual Blockage with Trastuzumab plus Pertuzumab}

In the phase II NeoSphere trial [27], 417 patients with operable, locally advanced or inflammatory HER2-positive breast cancer were randomly assigned 1:1:1:1 to receive 4 neoadjuvant cycles of: trastuzumab plus docetaxel (group A) or pertuzumab and trastuzumab plus docetaxel (group B) or pertuzumab and trastuzumab (group C) or pertuzumab plus docetaxel (group D). Patients given pertuzumab and trastuzumab plus docetaxel (group B) had a significantly improved pCR rate (45.8\%) compared with those given trastuzumab plus docetaxel (group A, 29\%). 24\% women given pertuzumab plus docetaxel (group D) had a pCR, as did $16.8 \%$ given pertuzumab and trastuzumab (group C).

In the phase II TRYPHAENA trial [28], designed as a cardiosecurity study, 225 patients with operable, locally advanced, or inflammatory breast cancer were randomized 1:1:1 to receive 6 neoadjuvant cycles (Arm A: FEC + trastuzumab + pertuzumab $\times 3 \rightarrow$ docetaxel + trastuzumab + pertuzumab $\times 3$; Arm B: FEC $\times 3 \rightarrow$ docetaxel + trastuzumab + pertuzumab $\times 3$; Arm C: docetaxel + carboplatin + trastuzumab + pertuzumab $\times 6$ ). Adjuvant therapy was given to complete 1 year of trastuzumab. pCR (defined as ypT0 ypN0) was achieved in 50.7\% (Arm A), 45.3\% (Arm B), and 51.9\% (Arm C) of patients. The pCR rate was higher in patients with HRnegative tumors compared with patients with HR-positive tumors.

These results strongly support the use of dual-HER2 blockade for neoadjuvant treatment of HER2-positive breast cancer patients. The combination of chemotherapy plus trastuzumab and pertuzumab had the highest probability of being the best treatment in terms of pCR, although there are no direct comparisons. The im- 
pact of the pCR [29] in HER2 tumors in terms of EFS is clear (hazard ratio $0.49,95 \%$ CI $0.33-0.71$ ), and this association was greater for patients with HR-negative disease (hazard ratio $0.29,95 \% \mathrm{CI}$ 0.24-0.36) (table 4).

\section{Conclusion}

Neoadjuvant treatment is the best approach in locally advanced tumors and in early stages of HER2-positive disease and TNBC. The pCR has an important role as a prognostic factor in these subtypes of breast cancer. The standard neoadjuvant treatment is chemotherapy based on anthracyclines and taxanes. In TNBC, the addition of carboplatin and substitution of paclitaxel by nab-pacli- taxel should be considered, although it is currently controversial. In HER2-positive tumors, the standard treatment is dual blockage with trastuzumab and pertuzumab combined with chemotherapy (with or without anthracyclines). In selected HR-positive/HER2negative tumors in postmenopausal women, hormone therapy with an AI could be another option, and the reduction in the expression of Ki-67 during hormonal treatment is a surrogate prognostic marker.

\section{Disclosure Statement}

The authors have no relationships to disclose.

\section{References}

1 Fisher B, Brown A, Mamounas E, et al: Effect of preoperative chemotherapy on local-regional disease in women with operable breast cancer: findings from $\mathrm{Na}$ tional Surgical Adjuvant Breast and Bowel Project B-18. J Clin Oncol 1998;16:2672-2685.

2 Bear HD, Anderson S, Smith RE, et al: Sequential preoperative or postoperative docetaxel added to preoperative doxorubicin plus cyclophosphamide for operable breast cancer: National Surgical Adjuvant Breast and Bowel Project Protocol B-27. J Clin Oncol 2006;24:2019-2027.

$\checkmark 3$ Rastogi P, Anderson SJ, Bear HD, et al: Preoperative chemotherapy: updates of National Surgical Adjuvant Breast and Bowel Project Protocols B-18 and B-27. J Clin Oncol 2008;26:778-785.

4 Mieog JS, van der Hage JA, van de Velde CJ: Preoperative chemotherapy for women with operable breast cancer. Cochrane Database Syst Rev 2007;CD005002.

5 Cordoba O, Carrillo-Guivernau L, Reyero-Fernández C: Surgical management of breast cancer treated with neoadjuvant therapy. Breast Care 2018;13:DOI: $10.1159 / 000491760$.

6 NCCN guidelines version 4.2017. Invasive Breast Cancer.

7 Cortazar P, Zhang L, Untch M, et al: Pathological complete response and long-term clinical benefit in breast cancer: the CTNeBC pooled analysis. Lancet 2014;384: 164-172.

8 Symmans WF, Wei C, Gould R, et al: Long-term prognostic risk after neoadjuvant chemotherapy associated with residual cancer burden and breast cancer subtype. J Clin Oncol 2017;35:1049-1060.

9 Goncalves R, DeSchryver K, Ma C, et al: Development of a Ki-67-based clinical trial assay for neoadjuvant endocrine therapy response monitoring in breast cancer. Breast Cancer Res Treat 2017;165:355-364.

10 Dowsett M, Smitj I, Ebbs S, et al: Prognostic value of ki67 expression after short-term presurgical endocrine therapy for primary breast cancer. J Natl Cancer Inst 2007;99:167-170.

11 Robertson J: Peri-operative aromatase inhibitor treatment in determining or predicting long-term outcome in early breast cancer. The POETIC trial (CRUK/07/015). SABCS 2017; abstr GS1-03.

12 Ellis M, Summan V, Hoog J, et al: Randomized phase II neoadjuvant comparison between letrozol, anastrozol and exemestane for postmenopausal women with estrogen receptor-rich stage 2 to 3 breast cancer: clinical and biomarker outcomes and predictive value of the baseline PAM50-based Intrinsic subtype-ACOSOG Z1031. J Clin Oncol 2011;29:2342-2349.
13 Chia YH, Ellis MJ, Ma CX: Neoadjuvant endocrine therapy in primary breast cancer: indications and use as a research tool. Br J Cancer 2010;103:759-764.

14 Rapoport BL, Demetriou GS, Moodley SD, Benn CA: When and how do I use neoadjuvant chemotherapy for breast cancer? Curr Treat Options Oncol 2014;15: 86-98.

15 Rastogi P, Anderson SJ, Bear HD, et al: Preoperative chemotherapy: updates of National Surgical Adjuvant Breast and Bowel Project Protocols B-18 and B-27. J Clin Oncol 2017;26:13-15.

16 Von Minckwitz G, Schneeweiss A, Loibl S, et al: Neoadjuvant carboplatin in patients with triple-negative and HER2-positive early breast cancer (GeparSixto; GBG 66): a randomised phase 2 trial. Lancet Oncol 2014; 15:747-756

17 Sikov WM, Berry DA, Perou CM, et al: Impact of the addition of carboplatin and/or bevacizumab to neoadjuvant once-per-week paclitaxel followed by dosedense doxorubicin and cyclophosphamide on pathologic complete response rates in stage II to III triplenegative breast cancer: CALGB 40603 (Alliance). J Clin Oncol 2015;33:13-21.

18 Bear HD, Tang G, Rastogi P, et al: Neoadjuvant plus adjuvant bevacizumab in early breast cancer (NSABP B-40 (NRG Oncology)): secondary outcomes of a phase 3, randomised controlled trial. Lancet Oncol 2015;16:1037-1048.

19 Untch M, Jackisch C, Schneeweiss A, et al: Nab-paclitaxel versus solvent-based paclitaxel in neoadjuvant chemotherapy for early breast cancer (GeparSeptoGBG 69): a randomised, phase 3 trial. Lancet Oncol 2016;17:345-356.

20 Schneeweiss A, Jackisch C, Schmatloch S, et al: Survival analysis of the prospectively randomized phase III GeparSepto trial comparing neoadjuvant chemotherapy with weekly nab-paclitaxel with solvent-based paclitaxel followed by anthracycline/cyclophosphamide for patients with early breast cancer - GBG69. SABCS 2017; abstr GS3-05.

21 Gluz O, Nitz U, Liedtke C, et al: Comparison of neoadjuvant nab-paclitaxel+carboplatin vs nab-paclitaxel+gemcitabine in triple-negative breast cancer: randomized WSG-ADAPT-TN trial results. J Natl Cancer Inst 2018;110:628-637.
Slamon DJ, Eyland-Jones B, Stevenshak P, et al: Use of chemotherapy plus a monoclonal antibody against HER2 for metastatic breast cancer that overexpresses HER2. N Engl J Med 2001;344:783-792.

23 Gianni L, Eiermann W, Semiglazov V, et al: Neoadjuvant chemotherapy with trastuzumab followed by adjuvant trastuzumab versus neoadjuvant chemotherapy alone, in patients with HER2-positive locally advanced breast cancer (the NOAH trial): a randomised controlled superiority trial with a parallel HER2-negative cohort. Lancet 2010;375:377-384.

24 Buzdar AU, Suman VJ, Meric-Bernstam F, et al: Fluorouracil, epirubicin, and cyclophosphamide (FEC-75) followed by paclitaxel plus trastuzumab versus paclitaxel plus trastuzumab followed by FEC-75 plus trastuzumab as neoadjuvant treatment for patients with HER2-positive breast cancer (Z1041): a randomised, controlled, phase 3 trial. Lancet Oncol 2013;14:1317-1325.

25 Untch M, Loibl S, Bischoff J, et al: Lapatinib versus trastuzumab in combination with neoadjuvant anthracycline-taxane-based chemotherapy (GeparQuinto, GBG 44): a randomised phase 3 trial. Lancet Oncol 2012;13:135-144.

26 De Azambuja E, Holmes AP, Piccart-Gebhart M, et al: Lapatinib with trastuzumab for HER2-positive early breast cancer (NeoALTTO): survival outcomes of a randomised, open-label, multicentre, phase 3 trial and their association with pathological complete response. Lancet Oncol 2014;15:1137-1146.

27 Gianni L, Pienkowski T, Im YH, et al: Efficacy and safety of neoadjuvant pertuzumab and trastuzumab in women with locally advanced, inflammatory, or early HER2-positive breast cancer (NeoSphere): a randomised multicentre, open-label, phase 2 trial. Lancet Oncol 2012;13:25-32.

28 Schneeweiss A, Chia S, Hickish T, et al: Pertuzumab plus trastuzumab in combination with standard neoadjuvant anthracycline-containing and anthracycline-free chemotherapy regimens in patients with HER2-positive early breast cancer: randomized phase II cardiac safety study (TRYPHAENA). Ann 2013;24:2278-2284

29 Cortazar P, Zhang L, Untch M, et al: Pathological complete response and long-term clinical benefit in breast cancer: the CTNeoBC pooled analysis. Lancet 2014; 384:164-172. 